\title{
ESPESSURA DO MÚSCULO ADUTOR DO POLEGAR EM PACIENTES COM LESÃO RENAL AGUDA NA UNIDADE DE TERAPIA INTENSIVA
}

\author{
Layssa Pelegrino, Luis Felipe Aleixo, Bianca Depieri Balmant \\ Universidade do Oeste Paulista - UNOESTE, Curso de Nutrição, Presidente Prudente, SP. e-mail: \\ biancadepieribalmant@hotmail.com
}

\section{RESUMO}

Este estudo avaliou a espessura do músculo adutor do polegar (EMAP) e sua associação com estado nutricional e desfecho clínico de pacientes internados na Unidade de Terapia Intensiva (UTI) com Lesão Renal Aguda (LRA) em diálise. Para isto, foi realizada a mensuração da EMAP, aplicado a Avaliação Subjetiva Global (ASG), e coletado marcadores laboratoriais em 50 pacientes com diagnóstico de LRA em diálise recém internados na UTI. Adicionalmente, o desfecho clínico desses pacientes foi observado. Todos os pacientes apresentaram depleção do estado nutricional pela ASG. Observou-se uma relação estatisticamente significativa entre EMAP e mortalidade, bem como uma correlação entre EMAP e albumina e EMAP e creatinina. Conclui-se que o EMAP pode ser um preditor de mortalidade em pacientes com LRA em diálise na UTI.

Palavras-chave: diálise, mortalidade, avaliação nutricional, antropometria, estado nutricional.

\section{THICKNESS OF THE ADDUCTOR POLLICIS MUSCLE IN PATIENTS WITH ACUTE KIDNEY INJURY IN THE INTENSIVE CARE UNIT}

\begin{abstract}
This study evaluated the thickness of the adductor pollicis muscle (APMT) and its association with nutritional status and clinical outcome of patients admitted to the intensive care unit (ICU) with acute kidney injury (AKI) on dialysis. For this, the measurement of the APMT was performed, applied the Subjective Global Assessment (SGA), and laboratory markers were collected from 50 patients diagnosed with dialysis AKI recently admitted to the ICU. Additionally, the clinical outcome of these patients was observed. All patients had nutritional status depletion by SGA. There was a statistically significant relationship between APMT and mortality, as well as a correlation between APMT and albumin and APMT and creatinine. In conclusion, APMT may be a predictor of mortality in patients with dialysis AKI in the ICU. Keywords: dialysis, mortality, nutritional assessment, anthropometry, nutritional status.
\end{abstract}

\section{INTRODUÇÃO}

A lesão renal aguda (LRA) é caracterizada pelo rápido declínio da taxa de filtração glomerular (TFG), que é demonstrada clinicamente como um aumento significativo na creatina sérica e redução de sua eliminação na urina. Pode ser acompanhada também por acúmulo de produtos nitrogenados e distúrbios hidroeletrolíticos, responsáveis pelas manifestações clínicas heterogêneas dos pacientes $^{1,2}$. Por existir mais de 30 definições de LRA na literatura, um grupo multidisciplinar internacional (AKIN), propôs uma nova definição e classificação de lesão renal aguda, baseada na dosagem sérica da creatinina e no volume urinário, a fim de uniformizar este conceito para efeitos de estudos clínicos e principalmente, prevenir e facilitar o diagnóstico da doença ${ }^{3,4}$. 
A lesão renal aguda é uma das complicações mais constantes em Unidades de Terapia Intensiva (UTI), correspondendo de 20 a $40 \%$ das admissões hospitalares neste setor. Além disso, apresenta uma enorme taxa de mortalidade, variando entre $50 \%$ a $90 \%$ dos pacientes internados em UTI, sendo que dos pacientes sobreviventes, cerca de $20 \%$ necessita de diálise temporária ou permanente ${ }^{2}$. A literatura salienta que a lesão renal está associada a determinadas comorbidades, como Hipertensão Arterial Sistêmica (HAS), Diabetes Mellitus (DM), Doença Pulmonar Obstrutiva Crônica (DPOC) e cardiopatias ${ }^{4,5}$. Porém, estudos afirmam que em UTI, outras causas podem ser fatores precursores da LRA, podendo se desenvolver por meio da combinação de fatores como medicamentos, hipovolemia, sepse, hipotensão e doenças hemodinâmicas ${ }^{2,6}$.

Em pacientes com lesão renal aguda, é comum ocorrer aumento do catabolismo corporal, o que leva a um risco aumentado para o desenvolvimento de desnutrição ${ }^{7}$. Além disso, a perda aguda na função renal, leva a situações pró-oxidativas, pró-inflamatórias, hiperglicemia e até uma hipertrigliceridemia ${ }^{8}$. Desta forma, as alterações metabólicas ocorridas na LRA, como o aumento na secreção de hormônios catabólicos, leva a acidose e consequente diminuição da oferta de macro e micronutrientes pelo hipercatabolismo, apresentado clinicamente por uma diminuição significativa de massa magra ${ }^{7,8,9}$.

A avaliação do estado nutricional em pacientes portadores de LRA é um grande obstáculo, logo, a obtenção do diagnóstico nutricional também é afetada ${ }^{8,10}$. Parâmetros laboratoriais, como a albumina, pré-albumina e transferrina são utilizadas na prática clínica para observar a resposta metabólica do organismo. Entretanto, na LRA tais indicadores perdem sua precisão como marcador nutricional, uma vez que o estado inflamatório presente neste paciente favorece a queda de proteínas plasmáticas $^{8}$. Quanto ao exame físico e antropométrico, pacientes com LRA, podem ser consideravelmente afetados por retenção hídrica, sob sinais de uremia e modificações mentais ${ }^{7}$.

Atualmente, a Avaliação Subjetiva Global (ASG), tem sido bastante utilizada na prática clínica como método de avaliação nutricional, por abranger perguntas referentes à perda de peso, hábitos alimentares, sintomas gastrintestinais, capacidade funcional, stress da doença de base e exame físico ${ }^{11}$. No entanto, a literatura sugere que em pacientes com lesão renal, a utilização de uma única ferramenta de avaliação não é suficiente para o diagnóstico correto e confiável de desnutrição $0^{7,12}$.

Uma técnica de avaliação que vem sendo utilizada para avaliar desnutrição proteica, é a aferição da espessura do músculo adutor do polegar $(E M A P)^{13}$. Estudos utilizando EMAP, envolvendo pacientes clínicos ${ }^{14}$, hospitalizados ${ }^{15}$, cirróticos ${ }^{16}$, com acidente vascular cerebral $(\mathrm{AVC})^{17}$, cirúrgicos ${ }^{18}$, oncológicos ${ }^{19}$ e renal crônico em hemodiálise ${ }^{20}$, foram realizados, mas não há estudos que avaliem pacientes com lesão renal aguda em UTI. Portanto, não há ponto de corte definido para esta população.

Considerando que na LRA a desnutrição está relacionada à maior ocorrência de complicações, internação prolongada e mortalidade, a obtenção de um diagnóstico nutricional seguro para intervenção nutricional é de suma importância ${ }^{8}$. Entretanto, há poucos estudos na literatura que avaliaram o estado nutricional de pacientes com lesão renal aguda, e poucos dados prospectivos sobre 0 comportamento dos marcadores nutricionais no desfecho clínico de pacientes com lesão renal aguda em UTI.

Este estudo teve como objetivo verificar a associação da EMAP com estado nutricional avaliado pela ASG, e com a mortalidade de pacientes internados na UTI com lesão renal aguda em diálise.

\section{METODOLOGIA}

Estudo observacional longitudinal, com 50 pacientes internados em um hospital de alta complexidade do interior do Estado de São Paulo, com diagnóstico de lesão renal aguda.

Foram incluídos pacientes internados na UTI da instituição com diagnóstico de lesão renal aguda, segundo a classificação $\mathrm{AKIN}^{3}$, em tratamento hemodialítico, com mais de 18 anos, ambos os sexos, que concordaram em se submeter às avaliações e assinaram o Termo de Consentimento Livre Esclarecido (TCLE). $\mathrm{Na}$ impossibilidade do paciente em responder e assinar o TCLE, foi solicitado ao familiar ou responsável. Foram excluídos pacientes com doenças que pudessem exercer influência negativa no trofismo muscular (exceto desnutrição), doenças degenerativas, neurológicas ou motoras.

Os pacientes foram incluídos no estudo a partir do diagnóstico médico de lesão renal aguda 
e acompanhados até o fim da permanência na UTI, onde foram considerados alta ou óbito, para caracterização do desfecho clínico de interesse.

Os dados foram coletados após aprovação do Comitê de Ética para Pesquisa sob o número CAAE 98810718.4.0000.5515

\section{Caracterização Clínica}

Os dados de identificação, clínicos (diagnóstico) e bioquímicos (creatinina, ureia e albumina sérica) foram retirados do prontuário e do sistema eletrônico de pacientes. O tempo de internação na UTI, a evolução clínica e desfechos negativos como a mortalidade, foram obtidos também por meio de dados registrados no prontuário médico até o momento da alta hospitalar.

\section{Avaliação Nutricional}

A avaliação do estado nutricional e a antropometria, foram realizadas por pesquisadores treinados, que coletaram os dados para ASG e medida da EMAP.

Para avaliação da ASG, o paciente ou familiar respondeu às perguntas referentes à perda de peso, hábitos alimentares, sintomas gastrintestinais e capacidade funcional, para posterior classificação em nutridos (A), moderadamente desnutridos (B), ou desnutridos graves $(C)^{11}$.

A EMAP foi realizada com a face ventral da mão em repouso e o cotovelo em ângulo de aproximadamente $90^{\circ}$ sobre o membro inferior homolateral $^{21}$. Para pinçar o músculo adutor no vértice de um triângulo imaginário formado pela extensão do polegar e do indicador, foi utilizado o adipômetro Skinfold Caliper (marca Saehan ${ }^{\circledR}$ ), com escala de $0-60 \mathrm{~mm}$ e precisão de $\pm 1,0 \mathrm{~mm}$. 0 procedimento foi realizado em ambas as mãos por três vezes, sendo usado o valor médio como medida da $\mathrm{EMAP}^{13}$. Devido ao edema ocorrido em pacientes com lesão renal aguda, optou por utilizar o lado com menor edema para realização da EMAP.

\section{Análise dos Dados}

Para a análise de dados, foi utilizado o software Action Stat e o software R. Foram aplicados três testes, sendo o primeiro o teste de Sperman, para verificar a existência de correlação entre idade, dias na UTI, tempo de diálise, albumina, creatinina e ureia, para análise de regressão linear e identificação do grau de influência das variáveis estudadas sobre a EMAP.
O segundo teste realizado foi o teste de Odds Ratio, para verificar se as variações da EMAP influenciariam diretamente com a taxa de óbito dos pacientes. O terceiro teste foi o Exato de Fisher, para verificar a existência de associação entre variáveis qualitativas. $O$ valor de $p<0,05$ foi adotado como nível de significância estatística para todos os testes.

\section{RESULTADOS}

A amostra foi composta por 50 pacientes, com média de idade de $61 \pm 13,66$ anos, sendo a maior parte do sexo masculino (60\%). Estes pacientes apresentaram permanência média na UTI de 18 $\pm 13,61$ dias, sendo em média $7 \pm 5,12$ dias destinados ao tratamento hemodialítico. A Tabela 1 apresenta a caracterização clínica dos avaliados e a Tabela 2, alguns dados laboratoriais e antropométricos.

De acordo com a ASG, 94\% (47) dos pacientes foram classificados com desnutrição grave e $6 \%$ (3) com risco de desnutrição. Não houve pacientes eutróficos pela ASG.

Em relação à EMAP e mortalidade, percebe-se uma relação estaticamente significativa $(p=0,0261)$. A partir do ponto de corte definido pela curva ROC (Figura 1), e dos valores estimados pela regressão, foi possível encontrar o valor de corte da variável EMAP de $8,996481 \approx 9 \mathrm{~mm}$. Além disso, foi constatado por meio do teste de Odds Ratio $(O D=1,27)$, que conforme aumenta o resultado de EMAP, maior a chance de óbito em pacientes edemaciados. Pode-se dizer então, que a chance de o indivíduo ir a óbito, aumenta em $27 \%$ ao aumentar em 1 unidade da EMAP.

No que se refere à associação das variáveis, observou-se uma correlação negativa fraca entre EMAP e albumina $(r=-0,32)$ e correlação positiva fraca entre EMAP e creatinina $(r=0,34)$, significando que conforme aumenta EMAP, diminui os níveis de albumina sérica, assim como conforme aumenta EMAP, aumenta também os valores de creatinina. Observou-se ainda uma relação positiva forte entre tempo de diálise e tempo de internação na UTI $(r=0,79)$, significando que quanto maior o tempo de diálise do paciente, maior o tempo de internação na UTI. Não houve associação significativa entre ASG e $\operatorname{EMAP}(p=0,5707)$ 
Tabela 1. Características gerais de pacientes com lesão renal aguda em diálise, internados na UTI.

\begin{tabular}{l|lcc}
\hline \multicolumn{2}{c}{ Características } & $\mathbf{n = 5 0}$ & $\mathbf{\%}$ \\
\hline \multirow{2}{*}{ Sexo } & Feminino & 20 & 40 \\
& Masculino & 30 & 60 \\
\hline \multirow{4}{*}{ Faixa Etária } & $>20$ e $<40$ anos & 3 & 6 \\
& $>40$ e $<60$ anos & 15 & 30 \\
& $>60$ e $<70$ anos & 20 & 40 \\
& $>70$ e $<80$ anos & 7 & 14 \\
& $>80$ anos & 5 & 10 \\
\hline \multirow{5}{*}{ Comorbidades } & Hipertensão & 27 & 54 \\
Associadas & Diabetes & 11 & 22 \\
& Doenças Cardiovasculares & 18 & 36 \\
& Insuficiência Respiratória & 36 & 72 \\
& Infecção do Trato Urinário & 14 & 28 \\
\hline Via de Administração & Doenças Gastrointestinais & 8 & 16 \\
de Dieta & Neoplasias & 8 & 16 \\
\hline \multirow{3}{*}{ Desfecho Clínico } & Outros & 13 & 26 \\
& Sonda Nasoentérica & 2 & 4 \\
& Alta Hospitalar & 48 & 96 \\
\hline \multirow{5}{*}{} & Óbito & 10 & 20 \\
& Diálise Crônica & 37 & 74 \\
\hline
\end{tabular}

Tabela 2. Características laboratoriais e antropométricas de pacientes com lesão renal aguda em diálise, internados na UTI.

\begin{tabular}{l|rc}
\hline & & Média \pm DP \\
\hline \multirow{2}{*}{ Exames Laboratoriais } & Albumina & $2,32 \pm 0,56$ \\
& Creatinina & $4,25 \pm 1,63$ \\
& Ureia & $180,7 \pm 59,27$ \\
\hline \multirow{2}{*}{$\begin{array}{l}\text { Medidas } \\
\text { Antropométricas }\end{array}$} & Espessura do Músculo Adutor do Polegar & $14,35 \pm 6,46$ \\
& Mão Direita (n=14) & \\
& Espessura do Músculo Adutor do Polegar & $13,39 \pm 4,32$ \\
& Mão Esquerda (n=36) \\
\hline
\end{tabular}

\section{DISCUSSÃO}

Estudos revelam que a mortalidade em pacientes com LRA dentro da UTT ${ }^{5,6}$, está relacionada ao tardio diagnóstico de lesão renal aguda, idade avançada ${ }^{4}$, septicemia ${ }^{3,5,6}$, subsequente presença de mais comorbidades crônicas dos pacientes, associados há um prolongado tempo de hospitalização $0^{1,2,5}$, necessidade de diálise e possibilidade de progressão para a doença renal crônica ${ }^{4,6}$. No presente estudo, observou-se que a incidência de óbito foi elevada, porém ainda abaixo do encontrado na literatura, onde a taxa de mortalidade em pacientes com LRA em diálise na
UTI pode variar entre 80 a 90\%, prevalecendo em pacientes idosos ${ }^{1,2}$.

Os pacientes com LRA em diálise apresentam um déficit nutricional certamente influenciado pelo desfecho da doença ${ }^{3}$. Sabe-se que estes pacientes são hipercatabólicos e por isto, é de extrema importância tentar suprir suas necessidades calóricas e proteicas ${ }^{3,7}$. No estudo de Fiaccadori et al. ${ }^{22}$, a ASG apresentou maior prevalência de pacientes gravemente desnutridos, tendo correlação com o maior índice de mortalidade, independente da presença de comorbidades agudas, crônicas e complicações durante a internação. No presente estudo, 
também foi observado alta prevalência de pacientes desnutridos pela ASG.

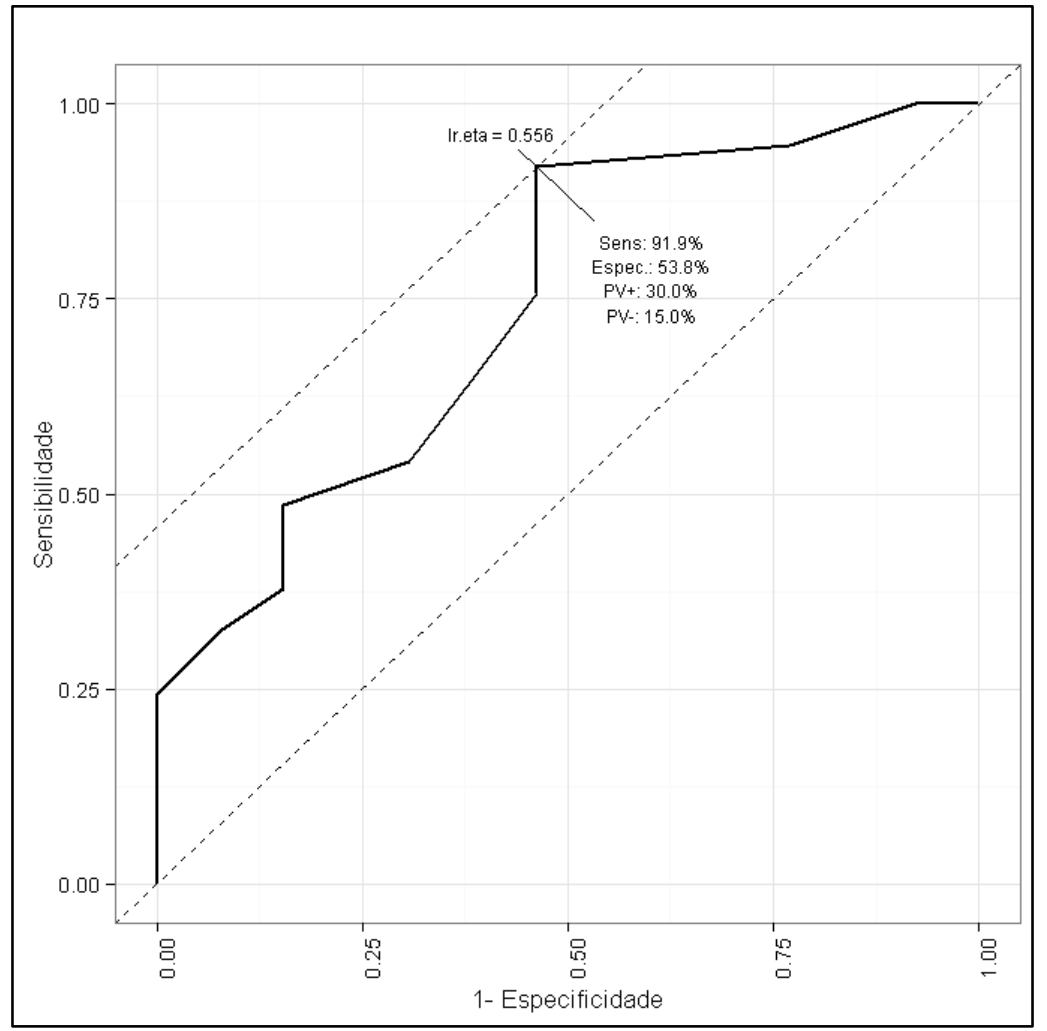

Figura 1. Curva de ROC para pacientes com lesão renal aguda em diálise, internados na Unidade de Terapia Intensiva, considerando a relação EMAP e mortalidade.

Embora haja estudos correlacionando a EMAP com baixa capacidade funcional e desnutrição ${ }^{21}$, o presente estudo mostrou que particularmente nos pacientes com LRA em diálise na UTI, o aumento da EMAP pode estar associado com mortalidade, devido à piora da função renal, principalmente relacionado ao edema e diminuição da albumina. Sabe-se que estados de edema e anasarca, comumente ocorridos em pacientes com doenças renais, pode comprometer medidas antropométricas, tornando essas medidas pouco fidedignas para acompanhamento nutricional ${ }^{8}$. Desta forma, o aumento da EMAP nesses pacientes não está relacionado ao aumento da espessura do músculo adutor, mas provavelmente ao edema presente, já que o aumento da EMAP está associado com diminuição da albumina e aumento da creatinina.

Ainda de acordo com os resultados apresentados, pacientes com LRA em diálise na UTI, com EMAP maior que 9, possuem maior chance de ir a óbito, sendo que ao aumentar 1 unidade da EMAP eleva em $27 \%$ a probabilidade destes pacientes virem a óbito. É valido ressaltar que a associação de condições clínicas como presença de edema, podem corroborar para esta predição. Ressalta-se ainda que estudos a cerca desta temática é escasso e por isto, devem ser estimulados.

Considera-se como viés do presente trabalho o tamanho amostral e coleta de dados clínicos. Desta forma, sugere-se que novos estudos, com amostras maiores e que associem dados clínicos referentes a função renal e estado clínico do paciente sejam realizados, a fim de melhor compreender a relação entre EMAP e mortalidade em pacientes com LRA em diálise na UTI.

Conclui-se que o aumento da EMAP pode ser um preditor de mortalidade em pacientes com LRA em diálise na UTI, por estar associado ao aumento da creatinina e diminuição da albumina. Entretanto, antes de sua utilização na prática clínica, orienta-se que esta relação seja melhor compreendida. 


\section{AGRADECIMENTOS}

À Universidade do Oeste Paulista UNOESTE pelo financiamento desta pesquisa.

\section{CONFLITO DE INTERESSES}

Os autores declaram não haver qualquer potencial de conflito de interesse que possa interferir na imparcialidade deste trabalho científico.

\section{REFERÊNCIAS}

1. Peres LAB, Wandeur V, Matsuo T. Preditores de injúria renal aguda e de mortalidade em uma Unidade de Terapia Intensiva. J Bras Nefrol. 2015;37:38-46.

2. Santos JCO, Mendonça MAO. Fatores predisponentes para lesão renal aguda em pacientes em estado crítico: revisão integrativa. Rev Soc Bras Clin Med. 2015;13:69-74.

3. Yu L, Santos BFC, Burdmann EA, Suassuna JHR, Batista PBP. Insuficiência renal aguda. Comitê de Insuficiência Renal Aguda da Sociedade Brasileira de Nefrologia. Diretrizes da AMB. 2007.

4. Luft J, Boes AA, Lazzari DD, Nascimento ERP, Busana JA, Canever BP. Lesão Renal Aguda em Unidade de Tratamento Intensivo: Características Clinicas e Desfechos. Cogitare Enferm. 2016;21:19. DOI: https://doi.org/10.5380/ce.v21i2.43822

5. Cerqueira DP, Tavares JR, Machado RC. Fatores preditivos da insuficiência renal e algoritmo de controle e tratamento. Rev Latino-Am Enferm. 2014;22(2):211-17.

DOI: https://doi.org/10.1590/0104-1169.3048.2404

6. Ávila MON, Rocha PN, Zanetta DMT, Yu L, Burdmann EA. Balanço hídrico, injúria renal aguda e mortalidade de pacientes em unidade de terapia intensiva. J Bras Nefrol. 2014;36(3):37988.

7. Martins C, Cuppari L, Avesani C, Gusmão MH. Terapia Nutricional no Paciente com Injúria Renal Aguda. Projeto Diretrizes da AMB/SBNPE/Nutrologia. Rev Assoc Med Bras. 2011;1:1-11.

8. Berbel MN, Pinto MPR, Ponce D, Balbi AL. Aspectos nutricionais na lesão renal aguda. Rev
Assoc Med Bras. 2011;57:600-6. DOI: https://doi.org/10.1590/S0104$\underline{42302011000500022}$

9. Mahan LK, Escott-Stump S, Raymond JL. Krause - Alimenotos, nutrição e dietoterapia. 13.ed. Rio de Janeiro: Elsevier; 2013.

10. Guedes JR, Silva ES, Carvalho ILN, Oliveira $M D$. Incidência e fatores predisponentes de insuficiência renal aguda em unidade de terapia intensiva. Cogitare Enferm. 2017;22(2):e49035. DOI: https://doi.org/10.5380/ce.v22i2.49035

11. Detsky AS, McLaughlin JR, Baker JP, Johnston $\mathrm{N}$, Whittaker S, Mendelson RA, et al. What is subjective global assessment of nutritional states? JPEN. 1987;11(1):8-13. DOI: https://doi.org/10.1177/014860718701100108.

12. Valente KP, Silva NMF, Faioli $A B$, Barreto MA, Moraes RAG, Guandalini VR. Espessura do músculo adutor do polegar na avaliação nutricional de pacientes cirúrgicos. Einstein. 2016;14(1):18-24.

DOI:

https://doi.org/10.1590/S1679-45082016A03596

13. Lameu EB, Gerude MF, Campos AC, Luiz RR. The thickness of the adductor pollicis muscle reflects the muscle compartment and may be used as a new anthropometric parameter for nutritional assessment. Curr Opin Clin Nutr Metab Care. 2004;7(3):293-301. DOI: https://doi.org/10.1097/00075197-200405000-

00009.

14. Andrade PV, Lameu EB. Espessura do músculo adutor do polegar: um novo índice prognóstico em pacientes clínicos. Rev Bras Nutr Clin. 2007;22(1):28-35.

15. Cobêro FE, Gomes MC, Silva AP, Bernardi JL, McLellans KC. A medida do músculo adutor do polegar está associada com indicadores antropométricos de avaliação de massa magra e de massa gorda em pacientes hospitalizados. Ver Soc Bras Alim Nutr. 2012;37(2):174-82. DOI: https://doi.org/10.4322/nutrire.2012.014

1. 16. Nunes FF, Fernandes SA, Bertolini CM, Rabito El, Gottschall CB. Avaliação nutricional do paciente cirrótico: comparação entre diversos métodos. Sci Med. 2012;22(1):12-7. 
17. Oliveira DR, Frangella VS. Músculo adutor do polegar e força de preensão palmar: potenciais métodos de avaliação nutricional em pacientes ambulatoriais com acidente vascular encefálico. Einstein. 2010;8(4):467-72. DOI: https://doi.org/10.1590/s1679-45082010ao1763

18. Bragagnolo R, Caporossi FS, Dock-Nascimento DB, Aguilar-Nascimento JE. Espessura do músculo adutor do polegar: um método rápido e confiável na avaliação nutricional de pacientes cirúrgicos. Rev Col Bras Cir. 2009;36(5):371-6. DOI: https://doi.org/10.1590/S0100-

\section{$\underline{69912009000500003}$}

19. Freitas BJ, Mesquita LC, Teive NJ, Souza SR. Antropometria clássica e músculo adutor do polegar na determinação do prognóstico nutricional em pacientes oncológicos. Rev Bras Cancerol. 2010;56(4):415-22.

20. Pereira RA, Caetano AL, Cuppari L, Kamimura MA. Espessura do músculo adutor do polegar como preditor da força de preensão manual nos pacientes em hemodiálise. J Bras Nefrol. 2013;35(3):177-84. DOI: https://doi.org/10.5935/0101-2800.20130029

21. Ghorabi S, Ardehali H, Amiri Z, Shariatpanahi $Z V$. Association of the adductor pollicis muscle thickness with clinical outcomes in intensive care unit patients. Nutr Clin Pract. 2016;31(4):523-6. DOI: https://doi.org/10.1177/0884533615621547

22. Fiaccadori $E$, Lombardi $M$, Leonardo S, Rotelli $\mathrm{CF}$, Tortorella G, Borghetti A. Prevalence and clinical outcome associated with preexisting malnutrition in acute renal failure: a prospective cohort study. J Am Soc Nephrol. 1999;10:581-93.

23. Pinto, MPR. Avaliação nutricional em pacientes com injúria renal aguda: identificação de fatores de risco associados à mortalidade. 2011. 75p. [Dissertação]. Mestrado na Faculdade de Medicina de Botucatu, Universidade Estadual Paulista, 2011.

24. Pinto, MPR. Fatores de risco nutricionais associados à mortalidade em pacientes com lesão renal aguda de acordo com os critérios do Acute Kidney Injury Network (AKIN). 2015. 105p. [Tese]
Doutorado na Faculdade de Medicina de Botucatu, Universidade Estadual Paulista, 2015. 